\title{
edCrumble, A Data-Enriched Visual Authoring Design Tool for Blended Learning
}

\author{
Laia Albó and Davinia Hernández-Leo
}

\begin{abstract}
This paper presents an evaluation of edCrumble, a blended learning authoring tool for teachers. The tool visually represents learning designs and integrates data analytics to scaffold teacher design decisions. In addition to assessing the usability of edCrumble using UMUX questionnaire, analyses of participant views on the advantages and disadvantages of the tool in comparison to traditional course planning approaches and participant-elicited factors that can facilitate or hinder adoption of the tool are presented. Three evaluation workshops were held with teachers of different backgrounds, educational stakeholders, and students. Data-gathering instruments included interviews and questionnaires to collect qualitative and quantitative data. Further, design artifacts resulting from the workshops were analysed. The evaluation shows that the tool possesses specific features that facilitate the representation of and support for designing blended learning, uncovers the factors that may promote or inhibit its adoption, exposes its connection with solving actual educational challenges, and reveals the strengths and weaknesses in the tool's usability.
\end{abstract}

Index Terms-Authoring tools, blended learning, data-driven support, instructor interfaces, learning design.

\section{INTRODUCTION}

$\mathrm{T}$ HE learning design (LD) field aims to support teachers in becoming learning designers, improving their teaching practices through evidence-based design decisions and by supporting the sharing and co-creation of learning designs among teacher communities [1], [2]. Teaching is increasingly viewed as a design science [1], [3]: teachers design the best conditions for learners to learn, selecting the most appropriate activities, resources and methodologies, often influenced by technological and cultural advancements [3]. Although teachers are expected to plan their courses in teams [4], the design process itself is often a lonesome task that takes place at smallscale and near-to-practice [3], [5], [6]. According to several authors [7]-[9], this design process entails going beyond intervention planning before implementation. It is a cyclic process that also includes tuning the design during the intervention and adapting it after assessing its effectiveness (redesign) [7]. Research and practice in LD seek to provide

Manuscript received August 3, 2019; revised January 13, 2020; date of current version November 3rd, 2020. This work was supported by the Spanish Ministry of Science (TIN2014-53199-C3-3-R and TIN2017-85179-C3-3-R), the Maria de Maeztu Units of Excellence Programme (MDM-2015-0502), RecerCaixa (CoT project) and the UPF PlaQUID2012. D.H.L acknowledges suitable textual, visual, and computational means to represent teaching practices, as well as the tools to manage and share them in order to support all phases of the LD cycle [2], [10]. Recently, the need to achieve LD goals has become increasingly apparent, as the complexity of educational designs has mounted significantly through the widespread use of technology in education, which presents both challenges and opportunities.

On the one hand, the use of internet-connected technology has allowed teachers to go beyond the use of traditional faceto-face instruction and adopt more complex scenarios that combine multiple teaching and learning modalities (mixing learning contexts and spaces, physical and digital tools, time settings as well as formal and informal structures) [11], [12]. These blended scenarios present several design challenges since their design complexity often hampers the reporting of welldocumented case studies, which frequently lack evidence on how pedagogy and/or technology influence learning outcomes, making the study of their effectiveness difficult [13], [14].

On the other hand, these new complex educational contexts provide opportunities for educators and researchers because they usually integrate technology that simplifies the automated collection of educational data during teaching and learning processes [15]. These contexts can then provide data-based evidence to improve the overall quality of the learning experience. Although learning analytics (LA) is the most familiar type of data collected from specific technological environments that allow educators to evaluate how students are learning within a learning context [16], there are more types of data available, less-explored, that may contribute to better design educational practices. These include community analytics - the metrics and patterns of design activity within a community of teachers and related stakeholders [15], [17]-, and design analytics, which are the metrics of design decisions and related aspects that inform learning designs [15].

The interaction between LA, community analytics and design analytics might have positive implications for teaching and learning practice as well as for research. First, design analytics can provide a framework for interpreting LA, while LA results can support future design iterations (redesign). Moreover, community analytics can offer pointers for

the support by ICREA under the ICREA Academia programme. (Corresponding author: Laia Albó).

The authors are with the Department of Information and Communication Technologies, Universitat Pompeu Fabra, Roc Boronat, 138, Barcelona, Spain (e-mail: laia.albo@upf.edu,davinia.hernandez-leo@upf.edu). 
inspiration during the design process and opportunities for the teacher community to make inquiries. Finally, LA can contribute to community analytics by providing information about the impact on learning settings created within a community [15]. Nevertheless, adopting this evidence-based education by teachers poses several challenges. These include the "need to adopt the necessary mindset, acquire new practices and be afforded the time and resources required and awarded for their efforts" [18] as well as methodological and ethical challenges [18]. Despite the variety of existing LD tools and representations of pedagogical practices [19]-[24], new approaches addressing the challenges and the opportunities of the complex blended learning educational practices are needed.

Accordingly, this paper presents and evaluates a LD authoring tool, edCrumble [25], which aims to support teachers in designing blended learning scenarios. The novelty of the tool is that it enables the representation of blended educational practices through a novel visual representation of a layeredtimeline that is informed and supported by two types of analytics: design and community analytics [15], [25], [26]. EdCrumble is also integrated into a social platform [21], [27] that allows teachers to share their designs among a community of educators both within and outside their own institutions. This paper is divided into seven sections, including the introduction. After a description of the authoring tool (Section II), we introduce the research questions for the tool's evaluation (Section III). The fourth section covers the methodology used and the fifth presents and discusses the findings of the research. Limitations and future work can be found in Section VI. Finally, Section VII presents the conclusions, including recommendations for researchers aiming to develop LD tools.

\section{The edCrumble Authoring ToOL}

\section{A. Design Principles of edCrumble}

Despite the potential affordances of the Learning Design (LD) approach [1], [10] and remarkable but limited cases of high levels of use [9], several researchers have identified a gap in the use of LD tools by teachers [28]-[33]. Specifically, the literature [28] identifies five first-order barriers regarding the adoption of LD goals by teachers, including: lack of institutional support, lack of adequate teacher training, time/workload factors, conceptual complexity of methods and tools, and adoption by their peers. Moreover, the authors also posited two second-order barriers: the use of information and communication technologies (ICTs) in teaching practice and teacher motivation. To address these obstacles, the same researchers outlined what teachers need from LD tools, including:

1. Flexibility

2. Support for reuse and adaptation of designs

3. Support for cooperation among teachers

4. Support for reflection

5. Ease of use

6. Saving time

7. Textual vs. graphical representation
8. Activating design thinking processes teachers are familiar with

In line with the last point, Bennett, Agostinho, and Lockyer [5] point out that "support tools have most potential to improve design decisions by engaging with the key influences that shape existing design practice". On the other hand, Kurvits et al. [37] evaluated several existing LD tools and proposed implementing the following design guidelines in order to increase teacher acceptance:

1. The use of simple visual language to represent its structure and components (artifacts, tasks, roles, workflows, activity types).

2. Ease of use, allowing for lightweight integration with common web tools used by teachers.

3. The availability for teachers to create sub-versions and remixes of validated "template scenarios."

4. The availability of semantic links to generic pedagogical scenarios with contextualized learning scenarios and ex-post-facto teaching and learning stories, together with learning analytics data gathered during the lesson implemented in accordance with the scenario.

EdCrumble has been conceptualized and developed following a co-creation process through participatory design workshops [34]. Furthermore, design decisions during the design and development process were made while taking into account most of the aforementioned design principles. Accordingly, edCrumble has been built on top of the LdShake platform [21], [27] to address two of the identified teacher needs (support for reuse and adaptation of designs and support for cooperation among teachers). This integration allows teachers to co-edit, share, remix, and comment on designs made by themselves or others within a teaching community, since the LdShake platform provides social network features [21], [27]. Moreover, as the literature reveals [35], sharing designs can have pedagogical benefits, such as improved student learning outcomes, as well as productivity benefits, e.g., less time spent on preparation resulting from the re-use of effective ideas by other educators. This also allows us to address another of the above-mentioned teacher needs: saving time. As Laurillard [36] argues, "we make progress faster if we can learn from each other, and especially if we can transfer proven pedagogical practice through cross-disciplinary collaboration."

\section{B. Visual Representation for Blended Learning Designs}

The theoretical basis of edCrumble relies on the goals of the LD field and the need for visual representations of LD that can visually highlight the design characteristics of blended learning, in order to create awareness of its design complexities. Previous research [38] led us to identify the challenges of blended learning design, as well as the need for an online tool that is able to both plan and visually represent such inherent complexity. Therefore, the visual representation of blended learning designs that edCrumble offers originates from the visual representation shown in Fig. 1 conceived in our previous research [38], [39]. The main element of technically implemented novel visual representation is a timeline that 


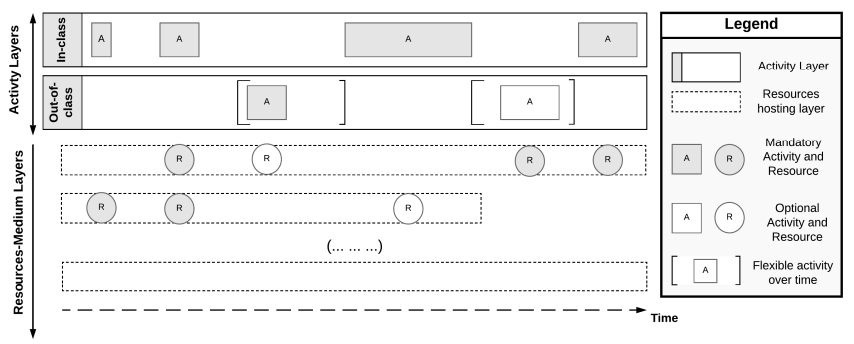

Fig. 1. Revised visual representation for blended learning designs.

comprises two activity layers (in-class and out-of-class). The granularity of the representation is variable, and is defined by the module's start and end dates, course, activity, etc. set by the teacher (as well as any potential time-lapse indicators, which could be weeks, days, etc.).

Below these two main layers are the resource layers, which are extensible and can be defined by the designer according to his or her needs. We may distinguish between the resource and the resource-medium layers, which show the medium whereby the resource is provided to students. For example, a book (resource) would fall into the physical resource-medium layer, much like other physical resources such as photocopies or laboratory materials; similarly, a Massive Open Online Course (MOOC) medium layer could contain a video, an online test or a web-text resource, among others. Thus, the activities are placed sequentially on the respective location where they take place within the timeline (in or outside of class), whereas resources are matched to the activities that use them and placed on the corresponding resource-medium layer, affecting if and when they are available (physical resource, online resource, virtual learning environment, web, cloud, etc.). Furthermore, blended activities and resources can be flagged as mandatory or optional. The blended learning visual model also defines how to represent the activities, mainly by using the following four descriptors:

1. Teacher presence (face-to-face, online, or not present).

2. Type of student work (individual, in groups, or whole class).

3. Type of task, following Bloom's taxonomy [40] (remembering, understanding, applying, analyzing, evaluating, and creating).

4. Grading mode (graded, not graded, or self-evaluation).

\section{EdCrumble Editor Analogy}

To date, several authors have employed different analogies to describe the underlying ideas of the LD approach. The theatre play comparison proposed by Koper and Olivier [41] in the IMS LD specification was used by Littlejohn and Pegler [14]. Here, the screenplay was the equivalent of a lesson plan, while the director's working document $-\mathrm{a}$ schematic of the technical performance with stage directions (choreography) and parallel processes (lighting, stage, directions, etc.) - was the activity sequence map [14]. Alternatively, Dalziel et al. [42] suggest similarities with a musical notation system to represent the abstract concept of music.
In line with the aforementioned ideas, we propose a novel visual analogy that corresponds to the characteristics underpinning the visual representation in which edCrumble is based (Fig. 1); the objective is to find an intuitive and userfriendly interface that already exists, in accordance with one of the teacher needs discussed in Section II-A. Observing the visual representation of blended learning designs [39], which are composed of different layers in time (Fig. 1), we believe that this could be compared to the process of working in layers when editing video. Similar to what happens in the blended visual representation, video editing uses several layers (containing mainly video and audio tracks) within a timeline (sequentially or in parallel) interconnected with resources (video transitions, effects, etc.) to generate the final video output. In this analogy, the output video (composed of a sequence of frames) would be equivalent to an educational practice (composed of a sequence of learning activities). This idea shares similarities and is in line with previous work in storyboarding LD representations where the timeline is the main element for the planning process (e.g. Carpe Diem [43]).

Furthermore, video editing is a clear example of how a complex process, which was until recently the exclusive domain of specialized professionals - as we might consider LD to be presently - has become available to anyone due to advances in technology and access (e.g., apps and tools that facilitate video editing by non-experts, including on smartphones).

\section{D. edCrumble Description}

Having covered the context and origins of the authoring tool, this section describes the functionalities of the main areas of the edCrumble editing interface (Fig. 2) [25]:

Context and general settings area: this allows users to provide general information about the LD such as the title, number of students, and start and end dates. It has three buttons to define: (1) the LD description, and the educational level and subject; (2) the list of learning objectives; and (3) the evaluation.

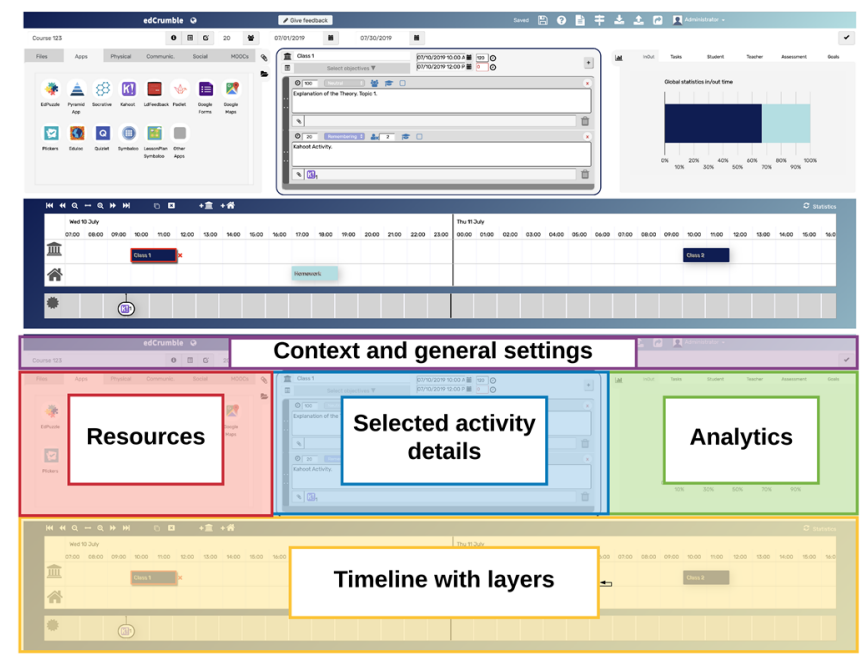

Fig. 2. EdCrumble editing interface areas. 
Timeline with layers area: this allows users to create in-class and out-of-class activities and place them in a timeline bounded by the dates entered in the context and general settings area. The timeline has two main layers by default (in- and out-of-class), where the activities are visualized sequentially depending on their schedule and type, as shown in Section II-B (see Fig. 1).

Selected activity details area: this allows users to edit the activities. Once an activity is selected, the user can define its learning objectives and add the tasks it includes. For each task, the following may be defined and edited: time allocated; associated cognitive process level, according to Bloom's taxonomy [40]; type of student work (individual, in groups, or whole class); teacher presence (teacher available face-to-face, online, or not present); and evaluation mode (graded, not graded, or self-evaluation). The user can also write a description of the task to be performed by the students with indicators for teachers and add the associated learning resources.

Resources area: this allows users to select the resources for the activities. Resources are divided into different categories, which appear as different tabs: Files, Apps, Physical, Communication, Social, and MOOCs. Users can drag and drop a resource to the task of an activity and edit its characteristics: title, description, target (teacher or student resource), hostmedium type (miscellanea, LMS, local storage, MOOC platform, web, physical artifact, cloud storage), and hostmedium name. Users can also specify a URL for the resource and/or upload a file. After adding a resource to an activity, a visualization of an icon associated with this resource automatically appears in the timeline, placed in a new layer depending on the host-medium type (see Fig. 2 where a resource added to the second activity's task in the selected activity area appears in grey in a host-medium layer within the timeline and aligns with the corresponding activity).

Analytics area: this allows users to consult design analytics, which are extracted from the meta-data of the produced LD itself. Design analytics are divided into different categories and appear as different tabs, Fig. 3 right upper corner): in-class/out-

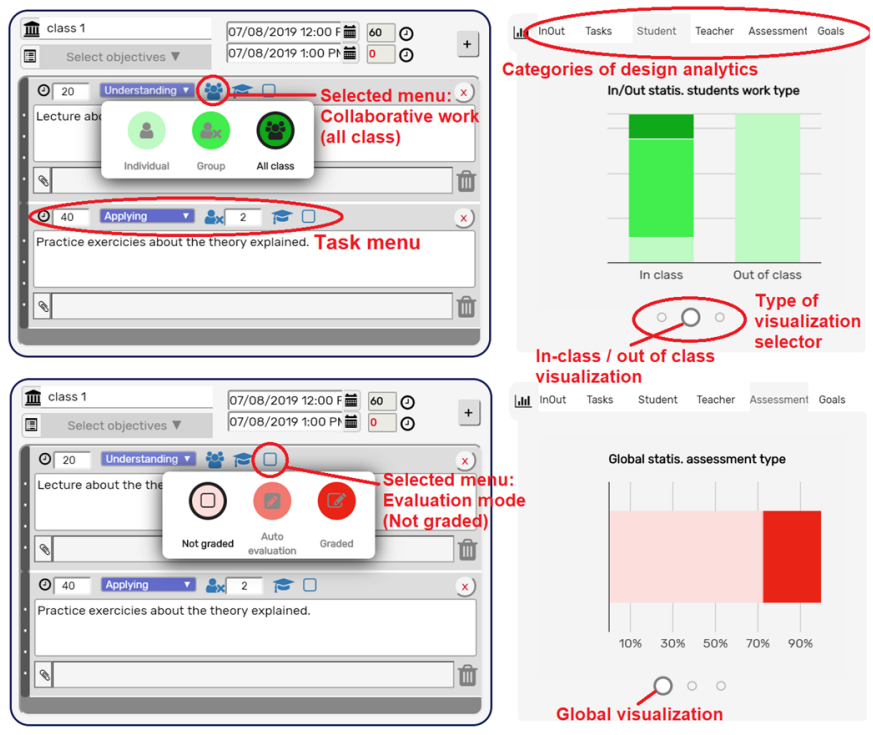

Fig. 3. Two screen captures of the Selected activity details and Analytics areas. Top: type of student work analytics with in/out class visualization. Bottom: evaluation mode analytics with the global visualization. of-class time analytics (DA1), task cognitive process (DA2), type of student work (DA3), teacher presence (DA4), and task evaluation mode (DA5). One may observe that the categories are the same as those in the task descriptors in the blended visualization described in Section II-B, as well as those in the selected activity area (each analytics category is identified by a different color).

Furthermore, each category has three different possible visualizations: global time statistics, statistics depending on the activity type (in- or out-of-class), and those depending on the learning objectives (Fig. 3). The global visualizations depict the time dedicated to each item in relation to the entire learning design (Fig. 3, lower left corner visualization), whereas the in/out class visualizations show the time for each category item separated into in- and out-of-class activity time (Fig. 3, upper right corner visualization).

Lastly, a button within the layered timeline area (Fig. 4, A and B), allows users to have another view of the timeline that hides the time intervals between the activities and shows the analytics per activity, which is controlled by a legend composed of buttons corresponding to the different LD analytics categories (Fig. 4, C to G). This results in a complete interactive visual representation of the LD.

The tool allows users to generate design analytics aggregated from all the LDs within a single folder, called "community analytics" [15]. It supports decision-making by teachers during the LD process not only individually but also at the community level by offering the possibility of considering LD analytics produced by other colleagues. Furthermore, edCrumble allows teachers to report the experience of applying the documented LD with their students. This feature is a text box where teachers can write their evaluations of the LD and provide a description of what worked well and what did not, so as to inform others who are willing to use their design. The text explanation is presented automatically in the online design summary, one of the tool's outputs.

\section{E. edCrumble Outputs}

EdCrumble outputs follow the approach of the LD_lite planning tool [14], a framework aimed to support educators' thinking through all the possibilities of course design in considering 'how to blend' [7]. LD_lite integrates three types of frameworks in one (the lesson plan, the pattern, and the sequence map). Similarly, edCrumble affords teachers the opportunity to generate four different output artifacts from the same design (a printable syllabus or lesson plan, an online design summary with a shareable URL, an embeddable interactive visualization, and a JSON-JavaScript Object Notation-file).

When researchers [7] asked a group of tutors about which framework from LD_lite was the most useful for describing and documenting practices and that could be most easily reused, their response was that, since all three types of descriptions serve different purposes and are useful to different people at different times, they were all useful aids for thinking through potential issues and designing blended learning [14]. Along these lines, Laurillard et al. [22, p. 10] suggest that "to represent 


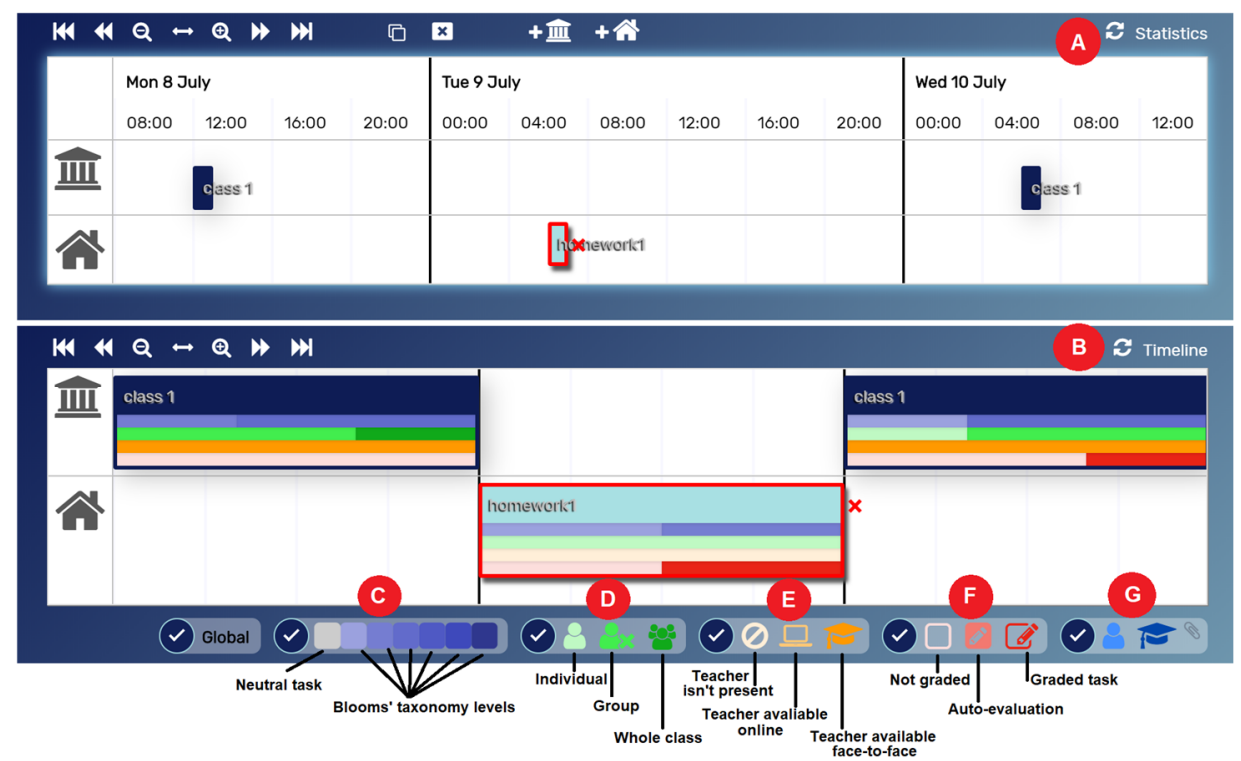

Fig. 4. Design analytics at the activity level. Top: timeline without the analytics. Bottom: timeline with the analytics (enabled using button A; disabled using button B). On the bottom, analytics menu C- F (design analytics categories), G (resource types: students vs. teachers).

fully the pedagogical properties of a learning design, it is important for the user to have access to multiple representations of the underlying properties of the domain model (given that a learning design plays out over time, we need to include a timebased representation as well)".

\section{$F$. Influence of existing $L D$ tools on the development of edCrumble and novel aspects}

Conole [44] distinguishes between two types of LD tools and representations: tools for visualizing designs (which can be used to visualize and represent LDs, e.g. LDVS [45]) and pedagogical planners (which can guide and support practitioners in making informed learning design decisions, e.g. Learning Designer [9] and Learning Design Studio [46]). Similarly to LePlanner [24], edCrumble has been conceived to fit into both categories bringing together the advantages of both types of tools, as well as providing data analytics to support the design process.

EdCrumble has been specifically devised bearing in mind the design challenges brought about by the complexities of blended learning designs. This allows teachers to visualize such complexity and to be aided during the design process by different types of data-analytics that highlight the blended characteristics. There are other tools that allow the design of blended learning scenarios (e.g. Learning Designer [9], LePlanner [24] and FROG [47]), and several tools that are based in the sequential representation of learning activities (e.g. LAMS [48] or FROG among others), some of them using a timeline (e.g. LePlanner) as well. However, to the best of our knowledge, there are no tools that consider the separation between in-class/out-of-class activities together with a visualization of the alignment with the resources and their delivery platforms within a timeline as edCrumble does. Furthermore, some LD tools provide dashboards with information about design characteristics (design analytics), e.g. the Learning Design Studio [46] or the Learning Design Support Environment (LDSE) [22] (which originally integrated the features of both the Learning Designer and the Course Resource Appraisal Model (CRAM) [49], a tool that enables users to analyze a course plan). Nevertheless, there is a lack of tools that combine the different types of analytics presented in the AL4LD framework [15]. A technically novel aspect of edCrumble is that it provides two types of analytics: design and community analytics.

Finally, the representation of the activities (following the boxes design approach with menus for characterizing each task within an activity) has been inspired mainly by the Learning Designer tool [9]. Despite having a similar activity area interface, however, edCrumble characterizes the tasks differently. Whereas the Learning Designer distinguishes between Read-Watch-Listen, Collaborate, Discuss, Investigate, Practice and Produce types of learning experiences [1] and uses Bloom's taxonomy to tag the learning outcomes, edCrumble categorizes the types of learning tasks based on Bloom's taxonomy and also allows matching the different learning outcomes with the described tasks. This decision was based on the challenges faced by users during edCrumble's codesign process using Laurillard's learning types taxonomy (e.g. some tasks could be described using more than one learning type simultaneously, which was impossible to do without first separating the descriptions in different boxes), as reported by Albó and Hernández-Leo [38]. On the contrary, Bloom's taxonomy is inclusive, in the sense that using a higher level implies the lower levels, avoiding the above issue. At the activity level, edCrumble has a few more differences with Learning Designer. edCrumble allows users to select educational resources from a resources panel, presents a new category for group size (whole class), and a new specification regarding teacher modalities (including teacher online).

\section{Research Goals Of The Evaluation}

Considering the aforementioned context in respect to LD tools, the objective of this study is to evaluate edCrumble based on 
the following topics stemming from the discussions within the previous section, which led us to formulate the corresponding research questions:

1. Design process support: study the extent to which the design tool can support and bring new perspectives to current teacher practices when designing blended courses. Specifically, to assess the general featureswhich can also be found in other LD tools - as well as the unique features that characterize edCrumble and blended learning representation and support, including the timeline, the design layers, and design analytics. RQ1: How can the tool (and its features) support the specific design process of creating blended learning designs?

2. Factors influencing tool adoption: explore what factors could promote or inhibit the adoption of the edCrumble authoring tool in actual practice. RQ2: What factors might be potential facilitators or disruptors of subsequent actual adoption of the tool?

3. Connection with the challenges of actual practice: to study the tool's potential for solving existing challenges in current teaching communities, since maximizing these connections and relevance could increase the probability of the tool's adoption. RQ3: What are the critical challenges (main points) of teachers that can be addressed by the tool and how?

4. System usability: determine whether the interface provided by the tool meets its usability objectives, in terms of maximizing ease of use, effectiveness, satisfaction, and efficiency. RQ4: How does the tool's user interface affect the perceived value of the tool?

\section{Methodology}

\section{A. Participants and Sample}

The study involved three groups of participants for the evaluation of the authoring tool. These three different groups were defined on the basis of their prior experience using the tool and the evaluation context.

Known teachers: there were 14 high school teachers from two different school communities who attended several participatory design workshops prior to this evaluation; in fact, they participated in the co-design process of the authoring tool. Participants were invited to join the last workshop (reported in this study) to evaluate the last version of the tool.

New teachers and edtech stakeholders: this group participated in a workshop held as part of a teaching innovation conference. It had 23 participants from different backgrounds, including teachers from different educational levels and edtechrelated stakeholders. Participants did not have any prior experience using edCrumble.

Undergraduate students: this group included 32 undergraduate students who participated in a local research study on blended learning at the authors' home university. They did not have any prior experience using edCrumble.

Whereas the 14 known teachers and the 23 participants (new teachers and edTech stakeholders) are the tool's target users, undergraduate students do not belong to this category. Their participation can be explained by the need to collect data for longer course designs - in this case, a three-month course - all the while assessing the tool's usability when introducing longterm designs, an overall much more complex task. Even though students were not able to provide meaningful contributions regarding the tool's pedagogical support because they are not the target users, they could provide valuable insights about the tool's usability. Students volunteered for the study and indicated 3 to 5 subjects from their bachelor's degrees on which they would be able to report. Researchers assigned one course per student depending on their preferences. Finally, students were granted a small financial compensation for using the system and participating in the study.

\section{B. Procedure}

Two types of workshops served as chances to interact with the system and give participants the opportunity to evaluate the tool. In the first type of workshop, the known teachers, new teachers, and edtech stakeholders had to document a given example of a short design (a module composed of two or three in-class sessions, and one or two out-of-class sessions). The 90minute workshop consisted of a role-play where participants were placed in groups of 2 to 4 . Each group of participants represented an imaginary school, while each participant in each group represented a teacher of a different subject (simulating different educational communities). The role-play had two main parts (individual and in group), both of which involved three steps. The individual activity (at an imaginary teacher-role level) consisted in:

1. Designing a short teaching unit with the online version of edCrumble; the researchers provided a printout of a ready-made design to each participant playing a teacher.

2. Analyzing the data resulting from the design.

Sharing the design created within the edCrumble community. The following group activity (at imaginary school-role level) involved:

1. Grouping several designs to generate community analytics.

2. Solving an educational challenge.

3. Discussing results with all participants.

The educational challenge involved using community analytics to balance the out-of-class workload between the different designs, which were part of a complete curriculum to be followed by the same student cohort (within the simulated school community). The goal was first to determine the total hours of homework given to the students by adding all the designs produced by the school; and second, if there were too many hours of out-of-class work, reduce them to a certain number deemed appropriate by debating which criteria to apply. At the end of the workshop, researchers asked the participants to fill in a research questionnaire to evaluate edCrumble. Finally, participants were asked to discuss in groups the educational problems that the tool can potentially solve as well as the factors that could either facilitate or hinder the habit of documenting with edCrumble. 
By contrast, in the second type of workshop, with undergraduate students, participants had to document their own design of a trimester-long course, having on average two/three in-class sessions per week over three months and all the out-ofclass activities and homework as well. The two-hour workshop consisted of the following phases:

1. 10 minutes: students signed the consent form and a document with their bank details (they received $€ 15$ as complementary compensation).

2. 15 minutes: researchers explained the aim and procedure of the study and made a short demonstration of how to document a course plan in edCrumble.

3. 80 minutes: students worked with edCrumble to enter the course plan into the system. Students were asked in advance to arrive sufficiently prepared to be able to document the course design assigned to each of them by the researchers.

4. 15 minutes: students filled out the evaluation questionnaire for edCrumble.

\section{Instruments, Data Collection, and Analysis}

Data were collected using two main instruments: semistructured interviews and a questionnaire. In addition, we used and analyzed the design artifacts resulting from the three workshops. Fig. 5 shows the instruments used in the data collection for each of the five evaluation topics (described in Section III): design process support, factors influencing tool adoption, connection with the challenges of actual practice, system usability, and learning design representation.

The interviews were conducted face to face and involved a series of open-ended questions that invited participants to share their perspectives regarding: their reflections on the possible advantages and disadvantages of using the tool as a new methodology; and teachers' views on visualizing and planning their lessons using a layered timeline (separating in- and out-ofclass activities). The interviews were conducted before the evaluation workshop and included only those participants who had already had experience with the tool (the known teachers' group). Due to teacher time constraints, the researchers were ultimately able to interview seven schoolteachers. The corresponding qualitative analysis of the responses focused mainly on identifying the advantages and disadvantages of

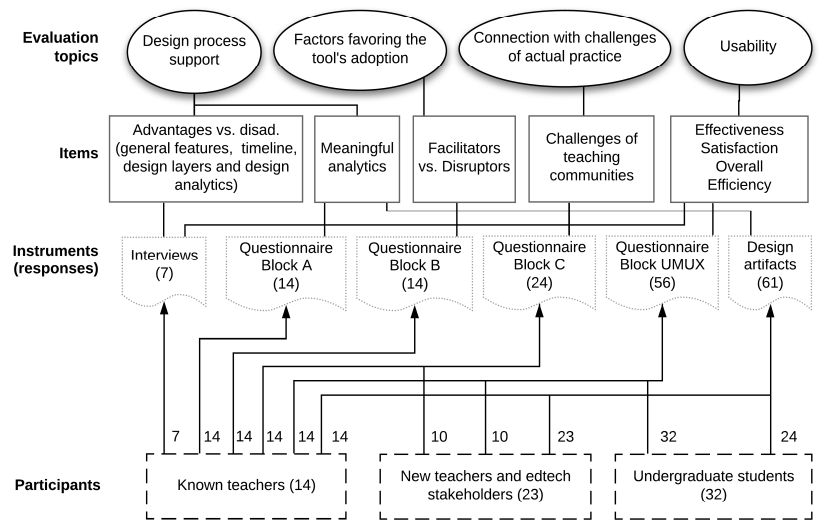

Fig. 5. Instruments used by evaluation topic and workshop participant. using edCrumble to evaluate the general features of the tool, as well as those designed specifically to support the blended learning design process (timeline, design layers, and design analytics). After classifying the advantages and disadvantages, we grouped the final items by related emerging topics, which led us to identify the general features in the advantages analysis and six emergent topics in the disadvantages analysis. We believe that the interview results provide a more in-depth analysis by the end users who had collaborated on the tool's concept and development from early on. This qualitative datawhich complements the quantitative data analysis performed on the questionnaire responses - therefore enriches our evaluation.

The research questionnaire consisted of four questions blocks: A, B, C, and UMUX. Block A complemented the design interviews regarding the assessment of the tool's design support, focusing specifically on evaluating design analytics. First, participants were shown three main statements and rated how much they agreed with each one on a five-point Likert scale. They could also provide additional comments or open responses for each statement. Second, participants were asked to evaluate design analytics based on several factors, once again on a five-point Likert scale.

Block B intended to discover which factors would promote the habit of documenting with edCrumble and which ones would hinder it instead. Researchers prepared (from the literature and previous work) 12 four-point Likert scale items that could facilitate tool adoption and three four-point Likert scale items that could discourage it. After rating all the proposed items, participants were provided with extra space at the end of the questionnaire to add new items for both types of factors.

Block C, participants were asked to use a five-point Likert scale to evaluate the level of importance of five educational challenges - taken from items selected by the researchers based on the literature and previous work-which could be addressed with the authoring tool. At the end of this block, participants were provided extra space to include any other relevant challenges that they believed the tool should/could resolve.

The fourth block focused on measuring the systems' user experience. Due to the limited workshop time, we decanted for the Usability Metric for User Experience (UMUX) questionnaire, a compact and highly reliable usability questionnaire. The UMUX instrument is a four-item Likert scale used for the subjective assess an application's perceived usability. It is designed to provide results similar to those obtained with the ten-item System Usability Scale, and follows the definition of usability established in ISO 9241-11 [50]. UMUX correlates with the SUS at a rate of $>0.80$ and is fully capable as a standalone subjective usability metric [50].

Finally, we analyzed the design artifacts resulting from the role-play workshop to check whether participants had been able to correctly generate the community analytics and whether they had successfully solved the proposed educational challenge, which helped to determine the pertinence of design analytics.

A reliability test was performed on the five items in block $\mathrm{C}$ of the questionnaire by calculating Cronbach's alpha. The results showed that the reliability of Block $\mathrm{C}$ was acceptable 
$($ Alpha $=0.96)$. Since blocks A and B were part of a designbased research iteration, their validity and reliability were ascertained by cross-referencing them with qualitative data (e.g. interviews, the open questions, etc.).

The researchers claim that data collection and analysis were conducted in accordance with all due ethical considerations, avoiding harm to participants, respecting their privacy through anonymous data collection, and ensuring that participation was voluntary; e.g., they could withdraw at any time without the need to provide a reason, and had the right to decline answering any question. At the beginning of the project, the researchers explained the context of the study and obtained the informed consent of all participants willing to take part in it.

\section{RESUlTS AND Discussion}

\section{A. Support During the Design Process}

1) Advantages and disadvantages: general features, timeline, design layers, and design analytics

In this section, we present the main findings from the interviews, which will help to determine whether the design tool can support and bring new perspectives to the practices teachers currently use to design their blended courses. Table I shows the advantages and disadvantages identified in teacher responses, divided into several categories. In terms of tool use, teachers deemed that increasing awareness, promoting reflection, and possibilities for sharing their ideas were three key advantages. They also stated that the system makes organizing the course easier and the day-to-day planning more systematic. Most participants agreed on the advantage of having everything planned with the tool automatically documented for later use, to be revised when redesigning the course and/or sharing it with other teachers. One teacher said that it would be very helpful to share the plan's visualization with the students in class, including access to the course resources.

Nevertheless, it is worth noting that the most frequently mentioned advantages were related to the tool's specific features to aid blended learning, which answers RQ1 as follows. First and foremost was the tool's timeline-based planning, which is especially important when designing a course for the first time, when everything must be planned from scratch. The second most highlighted advantage was the separation of inclass and out-of-class activity planning. One teacher said that this reminds us that the learning process happens not only inside the classroom, but also outside. Moreover, others believe that the tool allows teachers to be aware of how much time they require of students out of class, which is oftentimes not even considered when designing with other tools. Interestingly, they believe that the possibility of aggregating such data from different courses running in parallel could offer new insights in terms of teacher coordination, by sharing the type of work assigned to the students in a certain week to avoid repeating similar tasks or educational strategies, this further underscores one of the guiding principles for featuring community analytics in edCrumble. Finally, educators also mentioned the design analytics provided by the tool as advantageous in comparison to their traditional working habits, as well as the ability to print out the course syllabus from the planning generated with the tool, since some teachers prefer having their lesson plans on paper (e.g., for administrative purposes).

Conversely, a recurrent theme in the interviews was a sense among interviewees that the main disadvantage is the extra effort that tool entails. Documenting their practices with the tool translates into an extra workload that they often cannot afford. Sometimes it is a matter of priorities, where they feel that assessment work or other urgent day-to-day duties are more important than planning with the tool, which some teachers argue takes more time than using traditional planning methods. Furthermore, some teachers have been teaching a course for years and they already have their own organization methods, where everything has already been planned and the resources decided. Thus, despite the positive aspects, using edCrumble as a new method takes a considerable amount of effort that entails transferring their plans from their current system $/ \mathrm{s}$ into ours. In these cases, it is highly likely that they do not consider our system to be particularly cost-effective.

Some teachers also found the need to be very specific in terms of time allocation as a disadvantage when planning with edCrumble. Specifically, the requirement to specify the dates of the activities as well as the option to describe the duration of the tasks within the activities was seen as a constraint. In addition, another disadvantage is the lack of system flexibility, e.g., they would like to change their set plans easily and smoothly, even from a different device than a computer, such as through a smartphone app. Finally, the more experienced teachers commented that, although the tool provided good guidance and planning support for teachers who are just starting out, they would like it to feature more functions to re-adapt their already planned courses instead of focusing all the support on first-time planning.

Answering the first research question, the results presented above indicate that edCrumble supports teachers in the design of blended learning practices, especially with its timeline, design layers, and design analytics, as discussed in the above sections. The use of the tool can raise awareness, support reflection, as well as facilitate planning by providing the opportunity to share plans within a teaching community. Moreover, specific tool features - such as the timeline, the distinction between in-class/out-of-class layers, and the design analytics - have been deemed as advantageous over current teaching methods.

\section{2) Meaningful analytics}

Only half of the known teachers group responded to the first part of questionnaire's block A due to time constraints during the workshop. Despite the fact that we cannot draw strong conclusions from this small sample, we believe that it can provide qualitative insights to complement the evaluation of the analytics (together with the second part of the questionnaire, which was completed by 14 teachers).

As for the results, six out of seven respondents strongly agreed (with the seventh agreeing) with the following statement: "I think that the real-time visualizations while designing help me to better understand the design that I am 
TABLE I

Advantages ANd Disadvantages of Using OUR TOOl Compared to TRAditional TEacher Methods

(EXCERPTS TRANSLATED FROM THEIR ORIGINAL LANGUAGE)

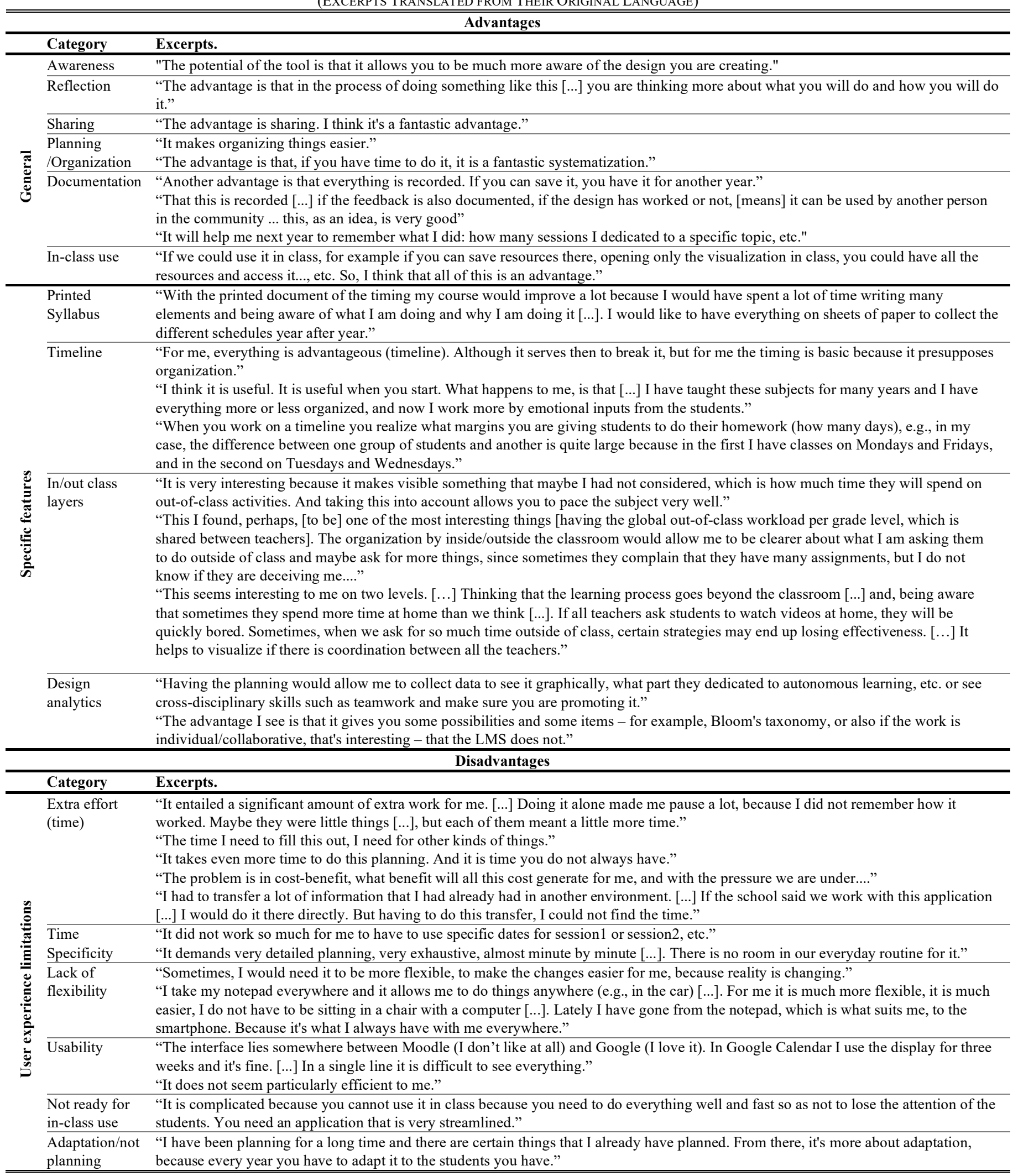

creating" (first statement in questionnaire block A). In the open responses, one participant stated that the visualizations would help him to time the distribution of activities more effectively.
Another participant highlighted the value of seeing how any activity is balanced pedagogically, while others mentioned that the analytics helped them to better organize themselves 
mentally, that it was very intuitive and helped them to stay aware of the design and the workload. It also allowed them to spot design errors and fix them. One participant added that the design analytics component is an outstanding feature of the learning design tool.

When participants were asked about whether they believe that their teaching practice would benefit from having design analytics for designs by other teachers within their teaching community (second statement in questionnaire block A), five participants strongly agreed, one agreed, and another one remained neutral. Most participants highlighted the value of sharing analytics as a positive aspect for improving, reflecting, and making objective design decisions, but there were discrepancies in whether analytics of designs from different educational backgrounds and topics than their own designs would also be useful. One participant suggested that it would be necessary to complement the design analytics with learning analytics to be able to discern the actual effect of edCrumble planning on student experience and outcomes. The last question asked participants whether they thought that their teaching practices would benefit from having design analytics of other teachers' designs from other teaching communities (third statement in questionnaire block A). The responses were also positive, with four participants agreeing strongly, two agreeing, and one remaining neutral. One participant stated that it would be a good opportunity to learn from brilliant teachers in other communities who have very interesting ideas that may be shared and applied.

In the second part of questionnaire block $\mathrm{A}$, the first factor under evaluation was the ease of interpretation, first for global visualization and then for in/out visualization. Most of the 14 participants strongly agreed that global visualization was easy to interpret (13 in the case of DA1, 8 in DA2, 12 in DA3 and DA4, and 11 in DA5). The others simply agreed and only two participants remained neutral (one in the case of DA2 and another in DA4). Only one person disagreed on how easy it was to interpret the global visualization on "type of knowledge" (DA2). Results for the in/out visualization were similar, with the conclusion that teachers found both visualizations, global and in/out, easy to interpret. The second factor studied was reflection support, the third was improvement support, and the fourth the potential of the design analytics categories to help in maintaining design continuity between in- and out-of-class activities. All the above-mentioned factors received similar positive evaluations from most participants in all the design analytics categories. The last factor evaluated, which applied only in category D1, asked participants about the out-of-class workload balance. Results were also positive, with 11 participants strongly agreeing with the statement that "edCrumble is very useful to help teachers ensure that the outof-class workload is not excessive," with a further two agreeing, and only one remaining neutral.

Lastly, regarding the artifacts collected after the role-play workshops, all participant groups were able to generate the community analytics with ease. Moreover, all groups successfully completed the challenge, being able to reduce the global out-of-class workload to within certain thresholds in each community. It is worth noting that participants used different strategies to overcome this challenge. While some groups agreed to reduce the same amount of out-of-class time for each individual design in the community, others only reduced the time of specific designs by, for instance, considering the nature of their subject, pedagogical strategy, or simply focusing on the design or designs that had assigned more out-of-class hours. Regarding the usefulness of having the aggregated design analytics for each course, participants argued that it has the potential to increase awareness of the work of other teachers within the same community. Above all, it allows teachers to coordinate different design strategies in order to offer students a more balanced workload.

In answer to the first research question, the results show that the design analytics component was a great aid for teachers in the design of blended learning, by fostering awareness during the design process, teacher coordination and collaboration, and workload balance between in- and out-of-class sessions, among others.

\section{B. Factors Favoring LD Tool Adoption}

Table II shows the results for factors that would promote the habit of documenting teaching practices with edCrumble. All 14 known teachers responded to block B of the research questionnaire in groups of two to three teachers. Thus, the total number of responses per item was six, corresponding to the number of groups formed.

Interestingly enough, the item that teachers considered to be potentially the most useful in terms of fostering the habit of documenting with the tool was option $\mathrm{f}$, global analytics at the community level. One possible use of the community analytics functionality could be the ability to monitor the number of outof-class hours per course for extra activities, as this is often a highly contentious topic between high school students and their teachers. When documenting several courses that run in parallel, the tool allows users to generate the aggregated time allocated for out-of-class activities, making visible the hours required both per course and in total. This allows teachers to balance the total time and adjust it depending on the needs of students and teachers alike, thereby avoiding overloading students with out-of-class work.

The next items by ranking were those related to: the opportunity to share designs between teachers in the same community (item d); the use of the tool for planning (i); the automatic generation of course syllabi from the planning $(\mathrm{k})$; and the flexibility to modify the planning on the fly (l). Teachers also stated that having pedagogical support integrated into the tool as well as its connection with other tools they usually use in their institution (e.g., their Learning Management System) could promote the habit of using edCrumble quite a lot (2 groups of teachers) or a lot (4 groups of teachers). These results are in line with the design guidelines proposed by Kurvits et al. [37]. Additionally, the tool's connection with other tools that specifically focus on planning (item $h$ ) was considered by 5 of the groups to be a potential facilitator, despite one group not considering it to be a facilitator at all. 
TABLE II

FACTORS PROMOTING THE HABIT OF DOCUMENTING WITH EDCRUMBLE (RESUltS IN FREQUENCIES)

\begin{tabular}{|c|c|c|c|c|}
\hline Likert Scales (1: not at all; 2: a bit, 3: quite a lot, 4: a lot) & 1 & 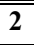 & 3 & 4 \\
\hline a. Pedagogical support integrated within the authoring tool. & 0 & 0 & 2 & 4 \\
\hline b. Resources support integrated within the authoring tool. & 0 & 1 & 2 & 3 \\
\hline c. That the authoring tool would facilitate the sharing of learning designs with teachers at other institutions. & 0 & 1 & 1 & 4 \\
\hline d. That the tool would facilitate the sharing of learning designs with teachers at the same institution. & 0 & 0 & 1 & 5 \\
\hline e. Mandatory use by the institution. & 3 & 1 & 1 & 1 \\
\hline f. Global analytics per grade level (community analytics) - e.g., homework balance control. & 0 & 0 & 0 & 6 \\
\hline g. Connection of the tool with existing tools used by the teachers in the institution (Clickedu, Moodle, etc.). & 0 & 0 & 2 & 4 \\
\hline h. Connection of the authoring tool with existing tools used for planning (Google Calendar, etc.). & 1 & 0 & 0 & 5 \\
\hline i. That the authoring tool would serve for planning. & 0 & 0 & 1 & 5 \\
\hline j. That the authoring tool would allow teachers to share the planning with students. & 0 & 1 & 1 & 4 \\
\hline k. That the tool would help me to generate the syllabus of the course (digitally or printed) automatically. & 0 & 0 & 1 & 5 \\
\hline $\begin{array}{l}\text { 1. That the authoring tool would allow me to document the changes I would make to the design easily after knowing what } \\
\text { happened in each class (e.g., using a mobile app that asks me how the class was and allows me to enter my feedback by voice } \\
\text { or text, for changes next year). }\end{array}$ & 0 & 0 & 1 & 5 \\
\hline
\end{tabular}

$\mathrm{N}=6$ groups of participants (14 participants divided into groups of 2 or 3 people).

Being able to share designs beyond their own institutions with teachers at other schools and sharing the planning with students ranked lower as facilitators compared to the abovementioned items. Although the results obtained were still positive - as most of the groups (4) thought that the tool would promote design sharing with other teachers from other institutions and with students "a lot" —one group indicated that it would do so "quite a lot" while another only "a bit". Similar results were obtained for the item on the resource support integrated into the authoring tool (see item $b$ ). The only factor that was considered to be definitely not a facilitator by the teachers was item e, which suggests that their institutions mandating use of the tool would not aid in its adoption.

Overall, these results indicate that aside from item e all the items identified by the researchers were correctly identified as facilitators, since most teachers thought that the factors under study would promote the habit of documenting with the tool quite a lot or a lot. These results match some of the teacher needs identified by Dagnino et al. [28], which are directly related to some of the identified factors. Furthermore, it is worth highlighting once again that, from a teacher's standpoint, the results indicate that the institution should not consider forcing them to use the tool if the long-term goal is to encourage its adoption. None of the teachers added any other factors to the list in the space provided for this purpose after block A. To date, 308 users have registered with edCrumble and one of the schools participating in its co-creation process has expressed their interest in adopting the tool at an institutional level.

Moving on to inhibiting factors, Table III presents the results for those that would hinder the habit of using the tool for documenting teaching practices. Consistent with the literature [28], [30] and our interview results, teachers' general lack of sufficient time and the delicate balance between the time necessary to "invest" in the tool versus the benefits obtained were identified by the participants as clearly disruptive factors, although there were different opinions regarding the lack of institutional recognition. Half of the teachers responded that this factor would not hinder acquiring the habit of using the tool for documenting their teaching practices at all, but the other half considered (at different levels) that not having institutional recognition for carrying out the task of documenting would definitely be a disruptive factor. Therefore, recognizing the time spent using design tools for documenting and sharing teaching practices could actually encourage some teachers to adopt these types of tools.

Finally, teachers also added their ideas about other disruptive factors. They mentioned the lack of systematics and the feeling of repeating already-completed tasks, as when entering their existing lesson plans into the tool, as two factors that would make acquiring the habit of documenting their teaching practices using edCrumble more difficult. On the one hand, we believe that the lack of systematics could be addressed at the institutional level by providing more support and reaching agreements with teachers on how to introduce new ways of working. On the other hand, the use of edCrumble as a planning tool from the beginning of the design process as well as improving its connection with tools that teachers are already using in their institutions could lower the perceived cost/benefit barrier in terms of time investment required. With respect to RQ2, the results presented above highlight several factors that may facilitate or disrupt subsequent adoption of the tool. Knowing which factors facilitate adoption may lead to improving the development process of future versions to yield a more essential design tool. The prioritization of future features will be based on selecting those which maximize facilitating factors while minimizing disruptors, which will allow the researchers to upgrade the tool with a view to wider adoption by teachers.

TABLE III

FACTORS IMPEDING THE HABIT OF DOCUMENTING WITH EDCRUMBLE (RESULTS IN FREQUENCIES)

\begin{tabular}{|c|c|c|c|c|}
\hline $\begin{array}{l}\text { Likert Scales } \\
\text { (1: not at all; } 2: \text { a bit, 3: quite a lot, } 4: \text { a lot }) \\
\end{array}$ & 1 & 2 & 3 & 4 \\
\hline a. Lack of time. & 0 & 0 & 0 & 6 \\
\hline b. Lack of institutional recognition. & 3 & 1 & 1 & 1 \\
\hline $\begin{array}{l}\text { c. Work where in the end the time that must be } \\
\text { invested vastly outweighs the benefits obtained. }\end{array}$ & 0 & 2 & 2 & 2 \\
\hline
\end{tabular}




\section{Connecting with Actual Practice Challenges}

This section evaluates the potential of the tool for solving existing challenges in current teaching communities. As mentioned in Section II, some of the challenges at the community level noted by several authors are the need to support reuse and adaptation of designs, as well as to foster cooperation among teachers [21], [27], [28]. Another challenge that we detected in previous studies during the co-creation of edCrumble with the known teachers' group was the need to monitor the workload of out-of-class activities, an issue that is related to the community analytics feature discussed in previous sections.

Table IV shows the perception results of the degree of importance of five items related to the aforementioned challenges. More than $60 \%$ of the participants rated the degree of importance of all five problems as level 4 or 5 , i.e. high or extremely high. The results from both groups of participants (known teachers, and new teachers and edtech stakeholders) were similar with no significant differences found. Items $b$ and c, regarding the exchange of teaching practices and resources among members of the same school, were the problems ranked highest $(92 \%$ and $88 \%$ respectively, rated as level 4 or 5 in terms of importance).

Thus, to answer RQ3, the teachers' highest priority is to address the lack of exchange practices and resources within their own community among teachers at the same institution. The second-highest area of need is to extend cooperation to other teaching communities beyond their own institutions. Lastly, there is the need to address the balance between in-class and out-of-class workloads. These results confirm that integrating of edCrumble with LdShake's community features is well justified. Moreover, it provides guidance for future work to prioritize the improvement of edCrumble's features related to solving the highest ranked problems, which could contribute to increasing the probability of the tool's adoption. As our previous research suggests [6], offering design tools that can address some of the teachers' day-to-day challenges may be a way of promoting the adoption of the LD approach since this can enhance the perception of the tool's utility.

\section{Usability Evaluation}

Table $\mathrm{V}$ provides the overall results obtained from the UMUX questionnaire $(\mathrm{N}=56)$. In answer to $\mathrm{RQ} 4,83.9 \%$ of the respondents were positive about the effectiveness of the system, agreeing that edCrumble's capabilities met their requirements. In terms of satisfaction, $76.8 \%$ disagreed that using the tool was a frustrating experience. As for overall impressions, $85.7 \%$ said that edCrumble was easy to use. The last research question in this study sought to determine the extent to which the tool's user interface affected its perceived value. Despite the positive perceptions of the effectiveness, satisfaction, and overall ease of use, it appears that the system's efficiency needs to be improved $30 \%$ of users think that they must spend too much time correcting things with the tool while $25 \%$ remained neutral), especially when designing long courses (the undergraduate group presented the most negative usability results since they had the most complex task which was to enter a design for a longer course).

TABLE $\mathrm{V}$

RESUlts (IN PERCENTAGeS) OF THE UMUX QUESTIONNAIRE MEASURING USER EXPERIENCE. LIKERT SCALE FROM 1 (STRONGLY DISAGREE) TO 7 (STRONGLY AGREE)

\begin{tabular}{|l|c|c|c|c|c|c|c|c|c|}
\hline \hline & $\begin{array}{l}\text { Strongly } \\
\text { disagree }\end{array}$ & & \multicolumn{4}{|r|}{$\begin{array}{r}\text { Strongly } \\
\text { agree }\end{array}$} \\
\hline & $\mathbf{1}$ & $\mathbf{2}$ & $\mathbf{3}$ & $\mathbf{4}$ & $\mathbf{5}$ & $\mathbf{6}$ & $\mathbf{7}$ \\
\hline $\begin{array}{l}\text { Effectiveness. The capabilities of } \\
\text { edCrumble meet my requirements. }\end{array}$ & 0 & 0 & 3.6 & 12.5 & 32.1 & 41.1 & 10.7 \\
\hline $\begin{array}{l}\text { Satisfaction. Using edCrumble is a } \\
\text { frustrating experience. }\end{array}$ & 28.6 & 30.4 & 17.9 & 14.3 & 7.1 & 1.8 & 0 \\
$\begin{array}{l}\text { Overall. edCrumble is easy to use. } \\
\text { Efficiency. I had to spend too much } \\
\text { time correcting things with } \\
\text { edCrumble. }\end{array}$ & 0 & 3.6 & 5.4 & 5.4 & 25.0 & 42.9 & 17.9 \\
\hline N=56. & 3.6 & 25.0 & 16.1 & 25.0 & 19.6 & 8.9 & 1.8 \\
\hline
\end{tabular}

\section{LIMITATIONS AND FUTURE WORK}

One of the main limitations of this study, much like other research in this field, is the limited number of teachers that participated in the study, compounded by the fact that they were volunteers who are motivated to continually improve their teaching practice. Most probably, these participants reflect a sub-set of "motivated" professionals who may not be representative of the wider population. Further studies are needed using a larger group of target users, particularly for the usability assessment. Although the 32 undergraduate students who participated in the evaluation provided useful insights in terms of technical usability, they were not target users in the tool's current development phase. Consequently, future work should include an extended usability study of an updated version of the tool, incorporating the improvements identified in this study. Furthermore, the future study should use SUS usability questionnaire to overcome the current limitations of UMUX [51]. Another limitation is that the UMUX questionnaire provides a subjective evaluation of system

TABLE IV

TEACHERS' PERCEPTION OF DEgREE OF IMPORTANCE (1: MiN, 5:MAX) OF THE Following PROBLEMS TO SOLVE (RESUlts IN PERCENTAGES)

\begin{tabular}{|c|c|c|c|c|c|c|c|c|c|c|c|c|c|c|c|}
\hline Workshop types & \multicolumn{5}{|c|}{ Known teachers } & \multicolumn{5}{|c|}{$\begin{array}{c}\text { New teachers \& } \\
\text { edtech stakeholders }\end{array}$} & \multicolumn{5}{|c|}{ All participants } \\
\hline Likert Scales (1: $\min , 5: \max )$ & 1 & 2 & 3 & 4 & 5 & 1 & 2 & 3 & 4 & 5 & 1 & 2 & 3 & 4 & 5 \\
\hline a. Balance between in-class and out-of-class workloads. & 7 & 0 & 29 & 14 & 50 & 0 & 20 & 20 & 30 & 30 & 4 & 8 & 25 & 21 & 42 \\
\hline b. Fostering the exchange of teaching practices among members of the same school. & 0 & 7 & 0 & 36 & 57 & 0 & 0 & 10 & 30 & 60 & 0 & 4 & 4 & 33 & 58 \\
\hline c. Fostering the exchange of teaching resources among members of the same school. & 0 & 0 & 14 & 36 & 50 & 10 & 0 & 0 & 50 & 40 & 4 & 0 & 8 & 42 & 46 \\
\hline d. Fostering the exchange of teaching practices beyond the same school. & 0 & 0 & 36 & 29 & 36 & 0 & 0 & 40 & 40 & 20 & 0 & 0 & 38 & 33 & 29 \\
\hline \multirow[t]{2}{*}{ e. Fostering the exchange of teaching resources beyond the same school. } & 0 & 7 & 21 & 29 & 43 & 0 & 0 & 40 & 30 & 30 & 0 & 4 & 29 & 29 & 38 \\
\hline & \multicolumn{5}{|c|}{$\mathrm{N}=10$} & \multicolumn{5}{|c|}{$\mathrm{N}=14$} & \multicolumn{5}{|c|}{$\mathrm{N}=24$} \\
\hline
\end{tabular}


usability. Future research should compare usability scoring with objective metrics, such as error rates and task timings, in a devoted experiment [50] to perform a more comprehensive usability assessment.

Overall, results indicate that future work is needed in order to improve the tool's UX, particularly focusing on features that would allow teachers to reduce the time and effort needed to document or introduce a design into the system. Most of the improvements have been reported by Albó and Hernández-Leo [39] and will be considered in future development iterations. Moreover, future studies should identify the factors that place undue demands on teachers' time and consider how the tool could be used to address them. Future work should also include new improvements that focus on making the interface even more intuitive and easy to use, so as to reduce the learning curve and scaffold the design process for new users who are not familiar with the tool. More research is needed to improve the current assessment types categories in the tasks menu (e.g. adding a peer-assessment category). Further research may also explore the connection of design and community analytics with learning analytics. The current version of edCrumble only provides a text box to report on the experience of implementing a design. There is a further need to automatize ways of connecting and visualizing the implemented LD's learning analytics, e.g., in the edCrumble timeline.

\section{CONCLUSION}

This article has described and evaluated edCrumble, a dataenriched visual learning design authoring tool for educators. The aim of edCrumble is to support the design and sharing of blended learning educational practices within a virtual community of educators. The evaluation has showed that the tool has specific features that facilitate support blended learning design: First, there is the timeline-based planning together with separate visual representations for in- and out-of-class activity planning; and second, the provision of design and community analytics, which facilitate teachers' awareness of the design characteristics and aid in their reflection process when planning complex blended-learning scenarios. Community analytics have also been identified as the most valuable feature that might act as a facilitator for the tool's future adoption by teachers. Other relevant aspects of the tool that might help increasing its usage are the opportunity to share designs between teachers and the tool's utility for planning. On the contrary, teachers' lack of time and the delicate balance between the time "invested" in the tool versus its benefits are still deemed as disruptive factors. Despite positive user perception in terms of the tool's effectiveness, satisfaction, and overall ease of use, results indicate that the system's efficiency needs to be improved.

Finally, our results have led us to formulate the following recommendations to those involved in the development of $\mathrm{LD}$ tools: provide design tools that address the lack of exchange practices and resources between teachers within the same institution, but also between teachers at different institutions; deliver tools that can address teachers' day-to-day needs and problems; offer time-based planning features; rely on the added value of being able to combine different types of analytics to better inform teachers about their design decisions. Our analysis also advises institutions against using a top-down approach on teachers (e.g. forcing them to adopt a specific LD tool), since this could backfire as an inhibitor for educators to adopt these kinds of tools. On the contrary, we recommend that institutions who are interested in adopting LD approaches explicitly acknowledge the importance of the learning design tasks performed by teachers (e.g. planning, redesigning and sharing) from the onset, allocating more time for teachers to focus on such tasks. Our tool is available online for free at https://ilde2.upf.edu/edcrumble/; all interested researchers and users are welcome to try it and send their feedback to help shape the tool's future development.

\section{ACKNOWLEDGMENT}

Authors would like to thank all the participants, collaborators and researchers who participated in the workshops.

\section{REFERENCES}

[1] D. Laurillard, Teaching as a design science: building pedagogical patterns for learning and technology. NY and London: Routledge, 2012.

[2] Y. Mor, B. Craft, and D. Hernández-Leo, "The art and science of learning design. Editorial," Research in Learning Technology, vol. 21, no. 22513, pp. 1-8, 2013.

[3] Y. Kali, S. Mckenney, and O. Sagy, "Teachers as designers of technology enhanced learning," Instructional Science, vol. 43, no. 2, pp. 173-179, 2015.

[4] Y. Kali, P. Goodyear, M. H. Ward, and L. Markauskaite, "Bridging multiple expertise in collaborative design for technology-enhanced learning," in Connecting Computer-Supported Collaborative Learning to Policy and Practice, CSCL2011 Conf. Proc. 2011, vol.2, pp. 831-835.

[5] S. Bennett, S. Agostinho, and L. Lockyer, "The process of designing for learning: understanding university teachers design work," Educational Technology Research and Development, vol. 65, no. 1, pp. 1-21, 2016.

[6] L. Albó and D. Hernández-leo, "Identifying design principles for learning design tools: the case of edCrumble," in 13th European Conference on Technology Enhanced Learning, EC-TEL 2018. 2018, pp. 406-411, doi: 10.1007/978-3-319-98572-5_31.

[7] D. Persico, F. Pozzi, and P. Goodyear, "Teachers as designers of TEL interventions," Br. J. Educ. Technol., vol. 49, no. 6, pp. 975-980, 2018.

[8] S. Bennett, L. Lockyer, and S. Agostinho, "Towards sustainable technology-enhanced innovation in higher education: Advancing learning design by understanding and supporting teacher design practice," Br. J. Educ. Technol., vol. 49, no. 6, pp. 1014-1026, 2018.

[9] D. Laurillard, E. Kennedy, P. Charlton, J. Wild, and D. Dimakopoulos, "Using technology to develop teachers as designers of TEL: Evaluating the learning designer," Br. J. Educ. Technol., vol. 49, no. 6, pp. 1044$1058,2018$.

[10] G. Conole and S. Wills, "Representing learning designs - making design explicit and shareable," Educational Media International, vol. 50, no. 1, pp. 24-38, 2013.

[11] R. A. Ellis and P. Goodyear, "Models of learning space: integrating research on space, place and learning in higher education," Review of Education, vol. 4, no. 2, pp. 149-191, 2016.

[12] A. Norberg, M. B. Stöckel, and M. Antti, "Time Shifting and Agile Time Boxes in Course Design," International Review of Research in Open and Distributed Learning: IRRODL, vol. 18, no. 6, 2017.

[13] G. Siemens, D. Gašević, and S. Dawson, Preparing for the digital university: a review of the history and current state of distance, blended, and online learning. Arlington: Link Research Lab, 2015.

[14] A. Littlejohn and C. Pegler, Preparing for blended e-Learning. London: Routledge, 2007.

[15] D. Hernández-Leo, R. Martinez-Maldonado, A. Pardo, J. A. MuñozCristóbal, and M. J. Rodríguez-Triana, "Analytics for learning design : A layered framework and tools," Br. J. Educ. Technol., vol. 51, no. 1, pp. 139-152, 2019.

[16] L. Lockyer and S. Dawson, "Learning designs and learning analytics," 
in Proc. 1st Int. Conf. Learning Analytics and Knowledge - LAK '11, 2011, pp. 153-156, doi: 10.1145/2090116.2090140.

[17] K. Michos and D. Hernández-Leo, "Supporting awareness in communities of learning design practice," Comput. Human Behav., vol. 85, pp. 255-270, 2018.

[18] S. McKenney and Y. Mor, "Supporting teachers in data-informed educational design," British journal of educational technology, vol. 46, no. 2, pp. 265-279, 2015.

[19] D. Persico, F. Pozzi, S. Anastopoulou, G. Conole, B. Craft, Y. Dimitriadis, D. Hernández-Leo, Y. Kali, Y. Mor, M. Pérez-Sanagustın, and H. Walmsley, "Learning design Rashomon $\mathrm{i}$ - Supporting the design of one lesson through different approaches," Res. Learn. Technol., vol. 21, 2013.

[20] L. P. Prieto, Y. Dimitriadis, B. Craft, D. Michael, V. Émin, M. Katsamani, D. Laurillard, E. Masterman, S. Retalis, and E. Villasclaras, "Learning design Rashomon II: Exploring one lesson through multiple tools," Res. Learn. Technol., vol. 21, pp. 1-20, 2013.

[21] D. Hernández-Leo, J.I. Asensio-Pérez, M. Derntl, F. Pozzi, J. Chacón, L.P. Prieto, and D. Persico, "An Integrated Environment for Learning Design," Frontiers in ICT, vol. 5, no. May, pp. 1-19, 2018

[22] D. Laurillard, P. Charlton, B. Craft, D. Dimakopoulos, D. Ljubojevic, G. Magoulas, E. Masterman, R. Pujadas, E.A. Whitley, and K. Whittlestone, "A constructionist learning environment for teachers to model learning designs," J. Comput. Assist. Learn., vol. 29, no. 1, pp. $15-30,2013$.

[23] S. Agostinho, "The use of a visual learning design representation to support the design process of teaching in higher education," Australasian Journal of Educational Technology.", vol. 27, no. 6, pp. 961-978, 2011.

[24] K. Pata, A. Beliaev, R. Robtsenkov, and M. Laanpere, "Affordances of the LePlanner for Sharing Digitally Enhanced Learning Scenarios," in Proc. IEEE 17th International Conference on Advanced Learning Technologies (ICALT), 2017, pp. 8-12, doi: 10.1109/ICALT.2017.77.

[25] L. Albó and D. Hernández-Leo, "edCrumble: designing for learning with data analytics," in 13th European Conference on Technology Enhanced Learning, EC-TEL 2018, 2018, pp. 605-608, doi: 10.1007/978-3-31998572-5 55.

[26] L. Albó, J. Barria-pineda, P. Brusilovsky, and D. Hernández-Leo, "Concept-Level Design Analytics for Blended Courses," in 14th European Conference on Technology Enhanced Learning, EC-TEL 2019, 2019, pp. 1-14, doi: 10.1007/978-3-030-29736-7 40

[27] D. Hernández-Leo, L. Romeo, M.A. Carralero, J. ChacóN, M. Carrió, P. Moreno, and J. Blat, "LdShake: Learning design solutions sharing and co-edition," Comput. Educ., vol. 57, no. 4, pp. 2249-2260, 2011.

[28] F. M. Dagnino, Y. A. Dimitriadis, J. I. Asensio-Pérez, and F. Pozzi, "Exploring teachers' needs and the existing barriers to the adoption of Learning Design methods and tools: A literature survey," Br. J. Educ. Technol., vol. 49, no. 6, pp. 998-1013, 2018.

[29] L. Cameron, "How learning design can illuminate teaching practice," Futur. Learn. Des. Conf., 2009.

[30] C. Lewin, S. Cranmer, and S. McNicol, "Developing digital pedagogy through learning design: An activity theory perspective," Br. J. Educ. Technol., vol. 0, no. 0, pp. 1-14, 2018.

[31] Y. Mor and R. Abdu, "Responsive learning design: Epistemic fluency and generative pedagogical practices," Br. J. Educ. Technol., vol. 0, no. 0 , pp. 1-12, 2018.

[32] D. Celik and G. D. Magoulas, "A Review, Timeline, and Categorization of Learning Design Tools," Adv. Web-Based Learn. - ICWL 2016. Lect. Notes Comput. Sci., vol. 10013, no. October, 2016.

[33] S. Bennett, S. Agostinho, and L. Lockyer, "Technology tools to support learning design: Implications derived from an investigation of university teachers' design practices," Comput. Educ., vol. 81, pp. 211-220, 2015.

[34] L. Albó and D. Hernández-Leo, "Co-creation process and challenges in the conceptualization and development of the edCrumble learning design tool," in Joint Proceedings of the CC-TEL 2018 and TACKLE 2018 Workshops, 2018.

[35] J. Dalziel, "Reflections on the Art and Science of Learning Design and the Larnaca Declaration," in The Art \& Science of Learning Design, M. Maina, B. Craft, and Y. Mor, Eds. Rotterdam, 2015, pp. 3-14.

[36] D. Laurillard, "The teacher as action researcher: Using technology to capture pedagogic form," Stud. High. Educ., vol. 33, no. 2, pp. 139-154, 2008.

[37] M. Kurvits, M. Laanpere, and T. Väljataga, "Analysis of Tools and Methods for Describing and Sharing Reusable Pedagogical Scenarios," in Advances in Web-Based Learning ICWL, 2015, pp.251-257, doi: 10.1007/978-3-319-25515-6_24.
[38] L. Albó and D. Hernández-Leo, "Conceptualising a visual representation model for MOOC-based blended learning designs," Australas. J. Educ. Technol., vol. 36, no. 2, pp. 1-26, 2019.

[39] L. Albó and D. Hernández-leo, "Co-creating a web-based visual representation model for authoring blended learning designs," Interact. Des. Archit., vol. 42, pp. 164-182, 2019.

[40] D. R. Krathwohl, "A revision of bloom's taxonomy: An overview," Theory Pract., vol. 41, no. 4, pp. 212-218, 2002.

[41] R. Koper and B. Olivier, "Representing th eLearning Design of Units of Learning," Educ. Technol. Soc., vol. 7, no. 3, pp. 97-111, 2004.

[42] J. Dalziel, G. Conole, S. Wills, S. Walker, S. Bennett, E. Dobozy, L. Cameron, E. Badilescu-Buga, and M. Bower, "The Larnaca Declaration on Learning Design," J. Interact. Media Educ., vol. 1, no. 7, pp. 1-24, 2016.

[43] G. Salmon, and P. Wright, "Transforming future teaching through 'Carpe Diem' learning design." Education sciences, vol. 4, no. 1, pp. 5263, 2014.

[44] G. Conole, Designing for learning in an open world, vol. 4. Springer Science \& Business Media, 2012.

[45] S. Agostinho, B. Harper, R. Oliver, J. Hedberg, and S. Wills, "A Visual Learning Design Representation to facilitate dissemination and reuse of innovative pedagogical strategies in University Teaching," in Handbook of visual languages for instructional design: Theories and practices, IGI Global., 2008, pp. 380-393.

[46] N. Law, W. Y. Ling Li, and H. L. Y. Farias, "Learning Design Studio: a pedagogically grounded productivity and collaboration platform for learning design and analytics professionals," in Companion Proceedings 8th International Conference on Learning Analytics \& Knowledge (LAK18), 2018.

[47] S. Håklev, L. Faucon, J. Olsen, and P. Dillenbourg, "FFROG, A Tool to Author and Run Orchestration Graphs: Affordances and Tensions,"in 13th International Conference on Computer Supported Collaborative Learning (CSCL), 2019, pp. 1013-1016.

[48] J. Dalziel, "Implementing learning design: the Learning Activity Management System (LAMS)," 20th Annu. Conf. Australas. Soc. Comput. Learn. Tert. Educ. 2003, pp. 7-10.

[49] E. Kennedy, D. Laurillard, B. Horan, and P. Charlton, "Making meaningful decisions about time, workload and pedagogy in the digital age: the Course Resource Appraisal Model." Distance Education, vol. 36, no. 2, pp. 177-195, 2015.

[50] K. Finstad, "The usability metric for user experience," Interact. Comput., vol. 22, pp. 323-327, 2010.

[51] S. Borsci, S. Federici, S. Bacci, M. Gnaldi, and F. Bartolucci, "Assessing User Satisfaction in the Era of User Experience: Comparison of the SUS, UMUX, and UMUX-LITE as a Function of Product Experience," Int. J. Hum. Comput. Interact., vol. 31, no. 8, pp. 484-495, 2015.

Laia Albó received the B.Sc. in Audiovisual Systems Engineering from the Universitat Pompeu Fabra (UPF) in 2013 and the MS in Education and ICT (e-Learning) from the Universitat Oberta de Catalunya in 2014, Barcelona, Spain. She defended her doctoral thesis on 2019 at UPF within the TIDE research group, in which she is now working as a postdoctoral researcher. Her research interests include educational technologies, data-analytics for education, learning design, blended learning, and artificial intelligence in education.

Davinia Hernández-Leo received the MS and $\mathrm{PhD}$ degrees in telecommunications engineering from the University of Valladolid, in 2003 and 2007, respectively. She is Full professor, ICREA Academia Fellow and head of the TIDE research group at the ICT Department of Universitat Pompeu Fabra, Barcelona. Her research interests includes Learning Technologies, with emphasis in learning design technologies, CSCL, community platforms, data analytics, and architectures and devices for learning. 